\title{
Estimation of Glutathione Level in Second Trimester of Pregnancy without Complications
}

\author{
Dr. Balasubramanian $\mathrm{A}^{1}$, Dr. Birundha $\mathrm{S}^{2 *}$ \\ ${ }^{1}$ Assistant Professor of Biochemistry, Government Medical College, Omandurar Govt. Estate, Chennai -600002, India \\ ${ }^{2}$ Assistant Professor of Biochemistry, Government Medical College, Omandurar Govt. Estate, Chennai - 600002, India
}

DOI: $\underline{10.36348 / \text { SIJB.2019.v02i09.003 }}$

| Received: 20.09.2019| Accepted: 27.09.2019 | Published: 30.09 .2019

*Corresponding author: Dr. S. Birundha

\section{Abstract}

Pregnancy though a normal physiological process exposes the person to oxidative stress. Glutathione is a master antioxidant; it protects both mother and foetus from the damage of oxidative stress and free radicals. In the beginning of pregnancy, Glutathione protects essential functions of the growing and developing embryo by controlling cell differentiation, cell death and other essential functions in the developing embryo. Hence this study proposed the level of glutathione in the pregnancy. Aim: To study the glutathione level in pregnant women ( $2{ }^{\text {nd }}$ TRIMESTER $)$ and to compare with the non-pregnant woman. Materials \& Methods: 50 pregnant women ( $2^{\text {nd }}$ TRIMESTER) and 50 non-pregnant women attending OBG department in government medical college, chennai Specimen: whole blood with EDTA. Method: Glutathione is determined by dithiobis nitro benzoicacid Glutathione levels were measured using the dithiobisnitrobenzoic acid (DTNB). Results: It was found that the glutathione levels in second trimester of pregnancy were found to be less than non-pregnant women. Conclusion: The result shows that oxidative stress during pregnancy is responsible for the reduction in glutathione as there is demand for the antioxidant glutathione. This study can be used to reinforce the necessity for the supplementation with dietary antioxidants like glutathione, vitamin $\mathrm{C}$, vitamin $\mathrm{E}$ etc.

Keywords: Pregnancy, Glutathione, Dithobisnitrobenzoic acid (DTNB).

Copyright @ 2019: This is an open-access article distributed under the terms of the Creative Commons Attribution license which permits unrestricted use, distribution, and reproduction in any medium for non-commercial use (NonCommercial, or CC-BY-NC) provided the original author and source are credited.

\section{INTRODUCTION}

Pregnancy is a stressful condition in which many physiological and metabolic functions are altered to a considerable extent. Consequently remarkable events occur during this period for sustaining mother and developing the growth and maintenance of foetus[1]. Normal pregnancy is accompanied by a high metabolic demand and elevated requirements for tissue oxygen, which results in increased oxidative stress and antioxidant defences[2].

Oxidative stress may be defined as a condition where there is disturbance in the pro-oxidant antioxidant balance [3]. The cells have evolved a number of counter acting antioxidant defences. Free radical scavenging mechanisms includes enzymatic and non-enzymatic antioxidants which limit the cellular concentration of free radical and prevent excessive oxidative stress [4]. Under normal conditions a variety of antioxidant mechanisms serve to control this peroxidative process. Pregnancy also has an effect on maternal antioxidant enzyme activities [5].
Antioxidant and glutathione status play an important role in the healthy pregnancy. The role of glutathione in the development of the foetus and placenta is crucial [5]. Glutathione can control cell differentiation, proliferation and apoptosis which are essential functions in the developing embryo and also it plays an important role in the development of organs (organogenesis) and the embryo (embryogenesis). The levels of antioxidants gradually decrease with the progression of pregnancy from first to third trimester. Low glutathione level, free radical damage and oxidative stress can result in many pregnancy complications such as birth defects, abortion and miscarriages [6]. A high level of glutathione significantly decreases the incidence of birth defects and protects both the mother and foetus from the pregnancy complications. Glutathione also helps to regenerate the stores of other antioxidants like vitamin E, vitamin C, \& folic acid, which further protect both mother and foetus [7].

In recent years the role of decreasing glutathione is gaining importance as they are threat for 
the normal pregnancy. Certain biochemical indices are useful in assessing the progression of pregnancy [7]. Hence this present study was undertaken to assess the role of glutathione level in second trimester of normal pregnancy.

\section{MATERIALS \& METHODS}

The study comprises of 50 normal pregnant women $2^{\text {nd }}$ trimester attending for antenatal check up in the obstetrics\& gynaecology department at our medical college medical Chennai, 50 healthy non pregnant women as control ranging in age from 20-30 years.

The case history have been collected through detailed questionnaire and the subjects with obesity, diabetes mellitus under medication and untreated diabetes, alcoholic, severely anaemic $(<6.0 \mathrm{gm} \%$ of $\mathrm{Hb})$ and those suffering from any other systemic disorder were excluded from the study.

$3 \mathrm{ml}$ of blood was drawn by venipuncture and collected in an EDTA coated tube and glutathione levels were determined by the following procedure.
The other laboratory investigations like fasting blood sugar, urea, creatinine, lipid profile also done. Determination of Glutathione by Dithiobisnitrobenzoic acid (DTNB) Method [6].

\section{Procedure}

Principle: Virtually all of the non-protein sulfahydryl groups of RBCs are in the form of reduced GSH 5, 5'-Dithiobis (2-nitrobenzoic acid) (DTNB) is a disulfide chromogen that is readily reduced by sulfohydryl compounds to an intensively yellow compound. The absorbance of the reduced chromogen is measured at $412 \mathrm{~nm}$ and is directly proportional to the GSH concentration.

\section{AIM \& OBJECTIVES}

- To study the glutathione level in pregnant women and to compare with that of non-pregnant women.

- To assess the glutathione levels in the $2^{\text {nd }}$ trimester of the normal pregnant women as compared to nonpregnant women.

\section{RESULTS}

Table-1: Mean glutathione levels during second trimester of pregnancy

\begin{tabular}{|l|l|l|l|l|l|l|}
\hline Glutathione levels & $\mathbf{N}$ & Range & Minimum & Maximum & Mean & Std. Deviation \\
\hline Second trimester & 50 & 1.70 & 3.50 & 5.20 & 4.4300 & .42342 \\
\hline
\end{tabular}

Table-2: Mean glutathione levels among non-pregnant and pregnant second trimester

\begin{tabular}{|l|l|l|}
\hline Group & Mean Glutathione levels $\boldsymbol{\mu m o l e s} / \mathbf{g H b \%}$ & SD \\
\hline Pregnant $\left(2^{\text {nd }}\right.$ trimester $)$ & 4.43 & 0.42 \\
\hline Non-pregnant & 5.94 & 0.36 \\
\hline
\end{tabular}

\section{Box plot representation}

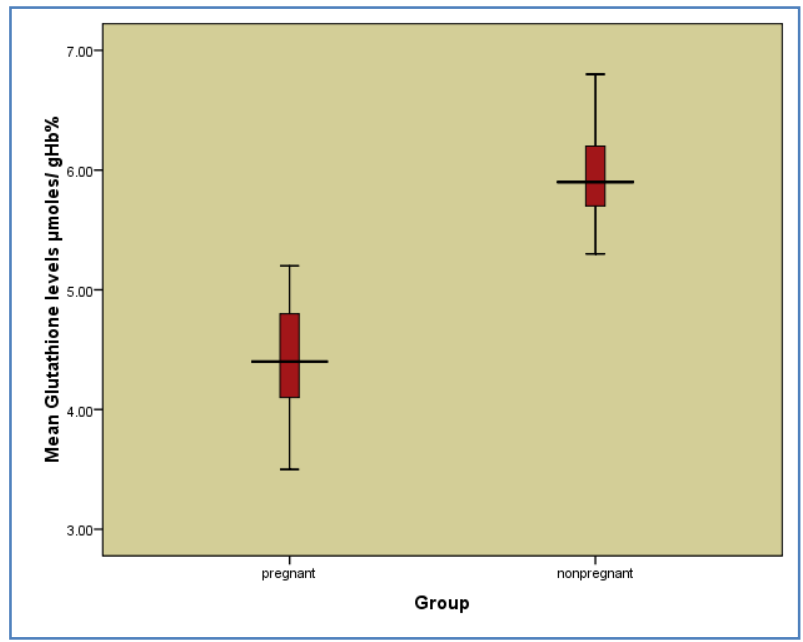

Fig-1: Comparison of mean glutathione levels among nonpregnant and pregnant second trimester

\section{PEARSONS CORRELATION}

The Pearson correlation between trimesters and the glutathione levels of pregnant women was statistically significant $-0.809, \mathrm{p}<0.0001$ meaning that with increasing duration of pregnancy the Glutathione levels decreased significantly.

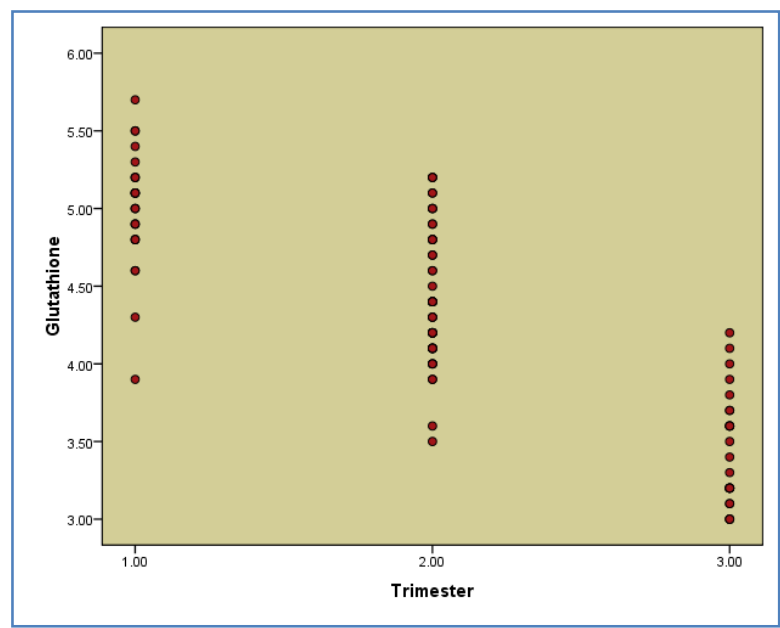

Fig-2: There was correlation between age of the woman and the Glutathione levels in the second trimester. (Pearson correlation $=0.287$, $p$ value $=\mathbf{0 . 0 4 3}$ ) 
Table-3: Comparison of Glutathione levels of pregnant and non-pregnant women

\begin{tabular}{|l|l|l|l|l|}
\hline Group & Mean Glutathione levels $\boldsymbol{\mu m o l e s} / \mathbf{g H b} \%$ & SD & t statistic & P value \\
\hline Pregnant $\left(2^{\text {nd }}\right.$ trimester $)$ & 4.43 & 0.42 & -19.179 & $<0.0001$ \\
\hline Non-pregnant & 5.94 & 0.36 & & \\
\hline
\end{tabular}

Serum glutathione levels are significantly lower in pregnant women than non-pregnant women. $(\mathrm{p}<0.0001)$

\section{DISCUSSION}

This is a case control study done on 100 study individuals (50 cases and 50 controls), in outpatient department of the obstetrics \& Gynaecology in Government medical college, omandurar estate, Chennai.

There are many studies showing the role of antioxidant in second trimester of pregnancy. This study showed the decrease in the level of antioxidants in pregnancy. This study also proved the significance of glutathione estimation (one of the major antioxidant) in pregnancy.

The results of the previous studies and this study showed that there was gradual decrease in glutathione levels from second trimester. In this study, it was found that $96 \%$ of pregnant women during second trimester had decreased glutathione levels and this difference was statistically significant. Hence, this study suggests the estimation of glutathione level during the second trimester of pregnancy.

The results of this study also supported by et $a l$. In the study they showed $80 \%$ decrease in glutathione level in second trimester.

This study and the previous studies shows the essential role of measuring glutathione during pregnancy in order to prevent the morbid complications like birth defects, abortion, miscarriages.

\section{CONCLUSION}

Overwhelming evidence indicates that antioxidants like glutathione, vitamin $\mathrm{C}$, vitamin $\mathrm{E}$ are crucial to all stages of pregnancy-from preconception to fetal growth and development, to labour and post-natal development [8].

Glutathione, as the master antioxidant, plays an important protective role in detoxifying these chemicals and reducing their damaging effects on the body.

Glutathione plays an important role in the development of organs (organogenesis) and the embryo (embryogenesis).

Glutathione help to protect the foetus from the damaging effects of pollutants, carcinogens and teratogens, and provide protection against the oxidative stress that is known to cause congenital malformations, Abortion and Miscarriage [9].

Numerous studies have shown that glutathione and other antioxidants are crucial in preventing oxidative stress in pregnant women with inflammation or disease conditions like diabetes and pre-eclampsia, or in foetuses at risk for developing cystic fibrosis.

GSH and antioxidant supplementation can decrease the incidence of birth defects and protect both mothers and the foetus from the damaging and possibly fatal consequences of pregnancy complications [10].

\section{REFERENCES}

1. Qanungo, S., \& Mukherjea, M. (2000). Ontogenic profile of some antioxidants and lipid peroxidation in human placental and fetal tissues. Molecular and cellular biochemistry, 215(1-2), 11-19.

2. Knapen, M. F., Zusterzeel, P. L., Peters, W. H., \& Steegers, E. A. (1999). Glutathione and glutathione-related enzymes in reproduction: a review. European Journal of Obstetrics \& Gynecology and Reproductive Biology, 82(2), 171184.

3. Granot, E., \& Kohen, R. (2004). Oxidative stress in childhood-in health and disease states. Clinical Nutrition, 23(1), 3-11.

4. Tiwari, A. K. M., Mahdi, A. A., Zahra, F., Chandyan, S., Srivastava, V. K., \& Negi, M. P. S. (2010). Evaluation of oxidative stress and antioxidant status in pregnant anemic women. Indian Journal of Clinical Biochemistry, 25(4), 411-418.

5. Sies, H. (1991). Oxidative stress: oxidants and antioxidants. Am j Med, 91:3c.

6. Walsh, S. W. (1997). The role-of oxidative stress and antioxidants in preeclampsia. Contemporary $o b$ gyn, 42, 113-124.

7. Biri, A., Kavuteum., Bozkurt, N., Devrim, E., Nurulu, N. (2006). Duraki JSOC Gynecol Investing, 13(5):384-8.

8. Kosower, N. S., \& Kosower, E. M. (1978). The glutathione status of cells. In International review of cytology (Vol. 54, pp. 109-160). Academic Press.

9. Meister, A. (1976). Glutathione metabolism and transport. Innygaard OF, SIMIC mg, edRadioprotectors and Anticarcinogens. New York, NY; Acedemic press.

10. Kidd, P. M., \& Huber, W. (1991). Living with the AIDS Virus: A Strategy for Long-term Survival. HK Biomedical, Incorporated, Education Division. 